\title{
The solar active region magnetic field and energetics
}

\author{
Qiang $\mathrm{Hu}^{1}$, Na Deng ${ }^{2}$, Debi P. Choudhary ${ }^{2}$, B. Dasgupta ${ }^{1}$, \\ \& Jiangtao $\mathrm{Su}^{3}$ \\ ${ }^{1}$ CSPAR, University of Alabama in Huntsville, Huntsville, AL, United States \\ email: qh0001@uah.edu \\ ${ }^{2}$ California State University Northridge, Northridge, CA, United States \\ ${ }^{3}$ National Astronomical Observatories, Beijing, China
}

\begin{abstract}
Motivated by increasingly more advanced solar observations, we recently develop a method of coronal magnetic field extrapolation, especially for an active region (sunspot region). Based on a more complex variational principle, the principle of minimum (energy) dissipation rate (MDR), we adopt and solve a more complex equation governing the coronal magnetic field that is non-force-free in general. We employ the vector magnetograms from multiple instruments, including Hinode, NSO, and HSOS, and particularly observations at both photospheric and chromospheric levels for one active region. We discuss our results in the context of quantitative characterization of active region magnetic energy and magnetic topology. These quantitative analyses aid in better understanding and developing prediction capability of the solar activity that is largely driven by the solar magnetic field.
\end{abstract}

Keywords. MHD, methods: data analysis, Sun: corona

\section{Motivation and Approach}

The magnetograph measurements of the solar magnetic field are probably the most direct and quantitative solar observations. The solar magnetic field plays a critical role in controlling solar activity that ultimately affects human life on Earth. There has been a long history of observing solar magnetic field, especially that in a solar active region (AR; sunspot region). Recent effort in ground-based and space-borne observations has yielded full vector magnetograms of solar ARs with modern instrumentations from, for instance, Huairou Solar Observing Station (HSOS), Big Bear Solar Observatory (BBSO), National Solar Observatory (NSO), Hinode, and Solar Dynamics Observatory (SDO). These observations provide magnetic field measurements on the solar surface at a limited number of heights, mostly on the photosphere only. Therefore the quantitative characterization of the coronal magnetic field largely depends on the numerical modeling, such as extrapolation (e.g., Wiegelmann 2010, this volume).

Solar coronal magnetic field extrapolation is to extrapolate the unknown coronal magnetic field in a finite volume from bottom boundary where the magnetogram provides the necessary boundary conditions. A common model of AR magnetic field is the force-free assumption that can be derived from the variational principle of minimum energy (Freidberg, 1987). We adopted an alternative variational principle, the principle of minimum (energy) dissipation rate (MDR), initially developed for fusion plasmas in the regime of magnetohydrodynamics (Montgomery \& Phillips, 1988; Dasgupta et al. 1998). Later it was extended to an open and driven system, like the solar corona, to establish a more complex governing equation of the coronal magnetic field (Bhattacharya et al. 2007). 
Bz_AR9661_ASP_ph11.txt

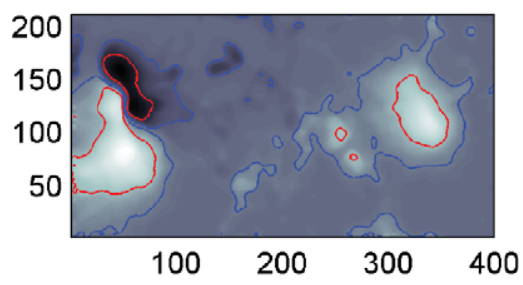

Bz_AR9661_ASP_ch11.txt

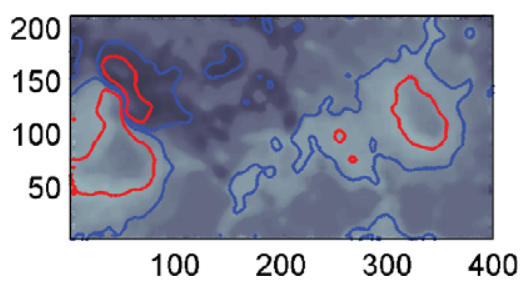

Bz0_AR9661_HS_large_0_1024.txt

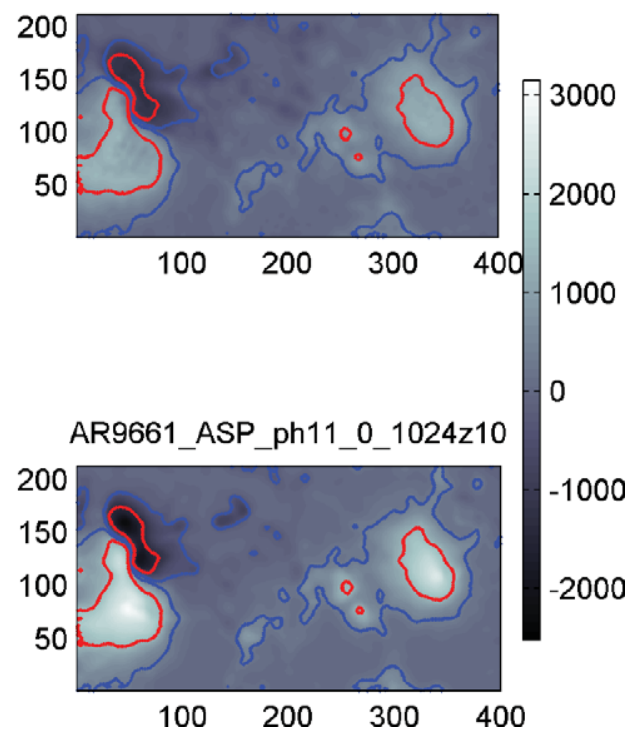

Figure 1. The $B_{z}$ component within the same field-of-view on the same gray scale in Gauss for AR 9661 on 17 Oct. 2001. Clock wise from the top left panel: ASP photospheric magnetogram, HSOS photospheric magnetogram, extrapolation result at $z \approx 611 \mathrm{~km}$, and ASP chromospheric magnetogram. The contours are $\left|B_{z}^{A S P}\right|=[300,1500] \mathrm{G}$.
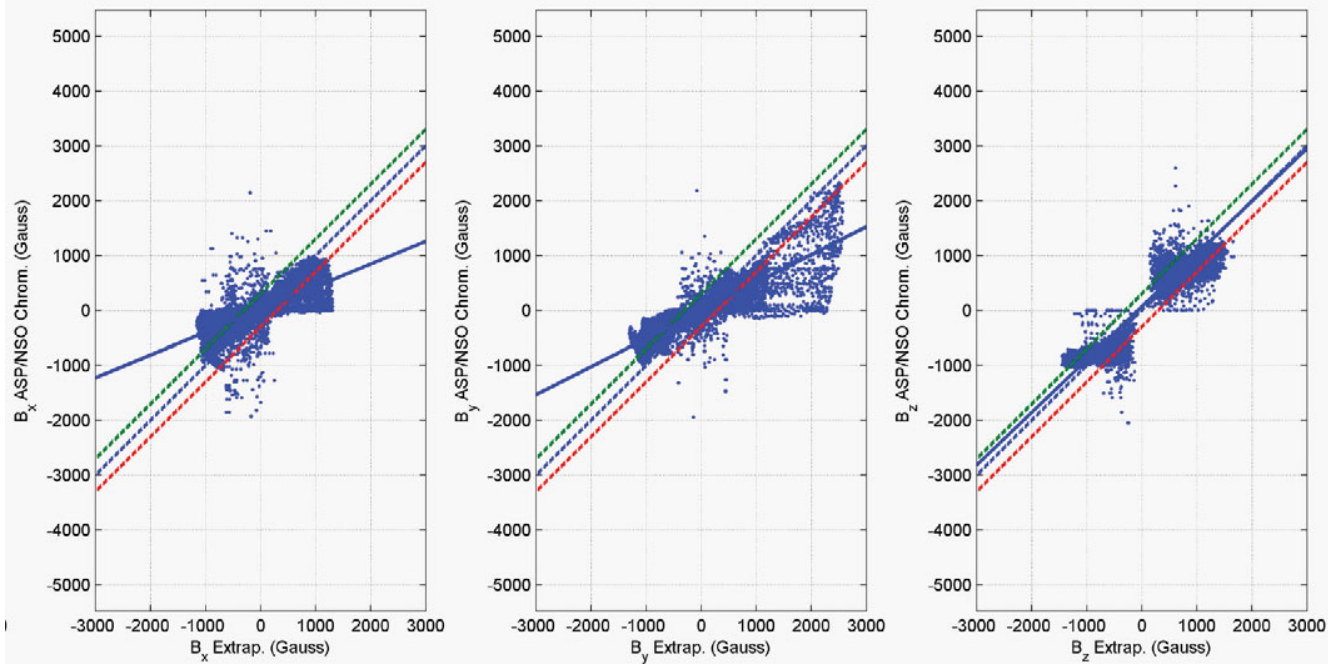

Figure 2. Component-wise comparison (from left to right: $x, y$, and $z$ component) of extrapolated (horizontal axis) and measured (vertical axis) magnetic field (in Gauss) at chromosphere.

Its solutions include force-free fields (e.g., Hu \& Dasgupta, 2008), but are more general, supporting non-vanishing plasma pressure gradient.

We devised an algorithm to extrapolate the coronal magnetic field into a Cartesian volume, utilizing measured vector magnetogram on the bottom boundary, based on the MDR theorem (Hu \& Dasgupta, 2006, 2008; Hu et al. 2008, 2010a,b). Ideally two layers 
of vector magnetograms at two heights are needed to construct the bottom boundary conditions ( $\mathrm{Hu}$ et al. 2008). An iterative procedure was recently developed to obtain the solution with one layer vector magnetogram (Hu et al. 2010a). The agreement between the measured and computed transverse magnetic field components on the bottom boundary is quantitatively assessed to judge in part the goodness of the solution.

\section{A case study: AR 9661}

We select AR 9661 to examine its magnetic field topology by the MDR-based extrapolation because a series of vector magnetograms and related observations are available. These data include both photospheric and chromospheric vector magnetograms from NSO Advanced Stokes Polarimeter (ASP) (Deng et al. 2010a), and vector magnetogram from HSOS, both on 17 Oct. 2001, 5 hours apart. Fig. 1 shows the multiple vertical component $B_{z}$ distribution within the same field-of-view on the same gray scale. There exists some variations for $300 \leqslant\left|B_{z}\right| \leqslant 1500 \mathrm{G}$ within which the ASP measurements are believed to be most reliable for both photosphere and chromosphere (Deng et al. 2010b). The HSOS measurements are generally smaller in magnitude than those of ASP. The chromospheric data (at a height $\sim 600 \mathrm{~km}$ ) are noisier and their magnitudes decrease by up to $1 / 3$ from photospheric values. The extrapolated magnetic field at a height $\sim 611$ $\mathrm{km}$ is generally greater in magnitude than observed, especially the transverse component, as further demonstrated in Fig. 2. The comparison is slightly improved upon a corresponding potential field extrapolation (not shown).

The extrapolated 3D field-line configurations are shown in Fig. 3 on grid $400 \times 212 \times 200$ for ASP photospheric data and $782 \times 715 \times 400$ for HSOS data, respectively. All have a grid size 0.42 " in each dimension, while the latter has a larger field-of-view. All configurations deviate significantly from the corresponding potential fields (not shown). Because of different computation domain and measurements on the bottom boundary, the magnetic field topology and connectivity are different. But significantly sheared low-lying arcades exist in the region of polarity inversion to the left in both plots. The total magnetic energy is $2.0 \times 10^{33} \mathrm{erg}$, and $2.2 \times 10^{33} \mathrm{erg}$, respectively.

Fig. 4, left panel, shows the MDI magnetogram with consecutive flare loop footpoints superimposed on 19 Oct. 2001 during a flare eruption (Qiu et al. 2009). The right panel shows the calculated squashing factor Q (Titov et al. 2002) of the extrapolated field from ASP photopheric magnetogram (Fig. 3 left panel). Although the flare occurred two days later, the flare loop footpoints seem to correspond to the large $\mathrm{Q}$ values near the polarity inversion region to the left where the flare initiated (Aulanier 2010, this volume). And they seem to match the root points of the corresponding sheared arcades in Fig. 3, especially those in the right panel.

\section{Discussion and Outlook}

All the extrapolations are carried out on $1024 \times 1024$ transverse grid. Better chromospheric data are needed to calculate the vertical gradient of the magnetic field over the whole domain. Clearly the finite computation domain and the boundary conditions greatly affect the extrapolation result and larger field-of-view is often more desirable (see also, DeRosa et al. 2009). More validation and applications combined with datadriven MHD simulation utilizing vector magnetograms are underway ( $\mathrm{Hu}$ et al. 2010b). In anticipation of the SDO full-disk vector magnetograms, it is desirable to extend the extrapolation to global scale. 

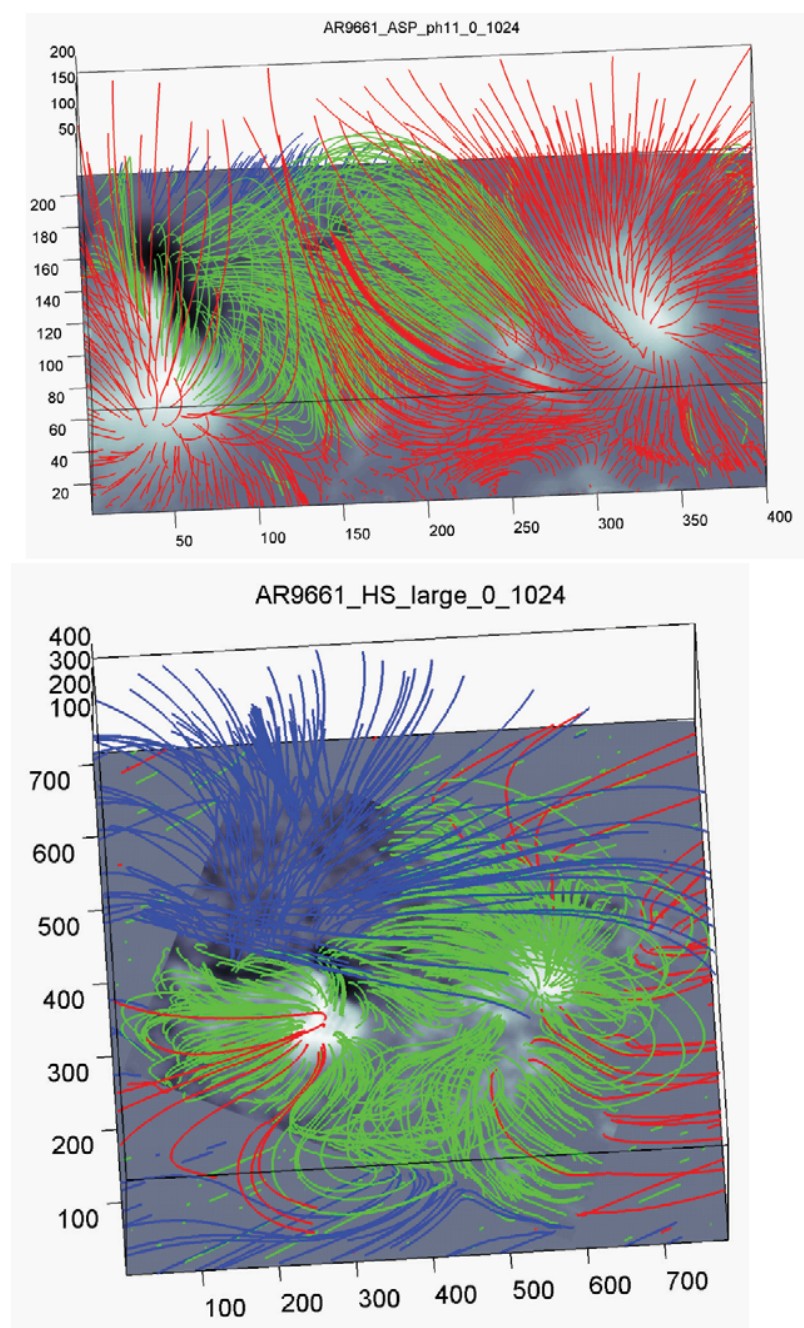

Figure 3. Extrapolated field lines from ASP photospheric vector magnetogram (left panel), and HSOS vector magnetogram (right panel). The bottom images are the $B_{z}$ distributions.
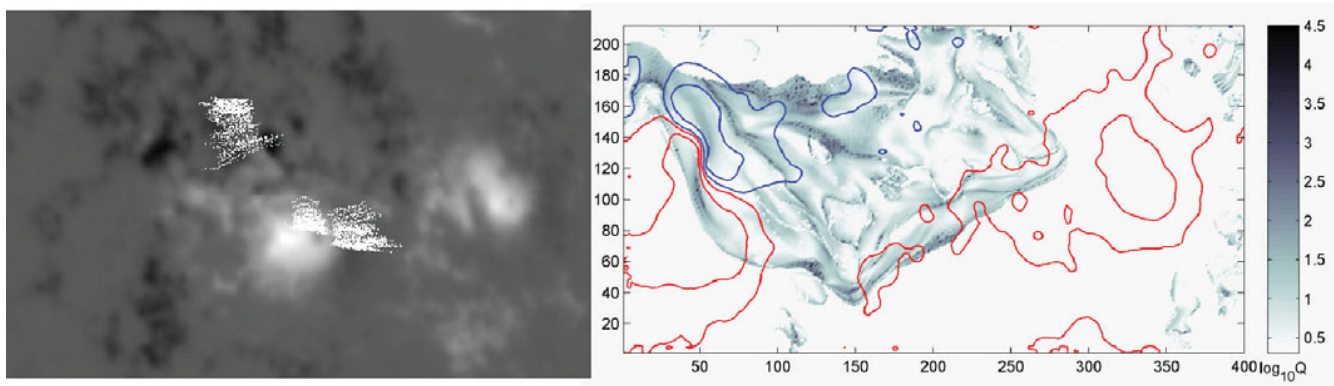

Figure 4. Left panel: the MDI magnetogram and flare loop footpoints (white dots) derived from TRACE images (courtesy of J. Qiu) on 19 Oct. 2001; Right panel: the squashing factor Q derived from Fig. 3, left panel. Contours are $B_{z}= \pm[300,1500] \mathrm{G}$. 


\section{Acknowledgements}

HQ and BD acknowledge NASA grants NNX07AO73G and NNX10AG03G for partial support. ND and DPC were supported by NASA grant NNX08AQ32G and NSF grant ATM 05-48260. HQ thanks Prof. J. Qiu for providing part of the plots.

\section{References}

Aulanier, G. 2010, this volume

Bhattacharyya, R., Janaki, M. S., Dasgupta, B., \& Zank, G. 2007, Solar Phys., 240, 63

Dasgupta, B., Dasgupta, P., Janaki, M. S., Watanabe T., \& Sato, T. 1998, Phys. Rev. Lett. 81, 3144

Deng, N., Choudhary, D. P., \& Balasubramaniam, K. S. 2010a, Astrophys. J., 719, 385

Deng, N., Choudhary, D. P., \& Balasubramaniam, K. S. 2010b, ASP Proceedings, submitted

DeRosa, M. L., et al. 2009, Astrophys. J., 696, 1780

Freidberg, J. P. 1987, Ideal Magnetohydrodynamics, Published by Plenum Press

Hu, Q., Wang, A., Wu, S. T., \& Gary, G. A. 2010b, Astrophys. J., submitted

Hu, Q., Dasgupta, B., DeRosa, M. L., Büchner, J., \& Gary, G. A. 2010a, J. Atmos. Sol. Terres. Phys. 72, 219

Hu, Q. \& Dasgupta, B. 2008, Solar Phys., 247, 87

Hu, Q., Dasgupta, B., Choudhary, D. P., \& Büchner, J. 2008, Astrophys. J., 679, 848

Hu, Q. \& Dasgupta, B. 2006, Geophysical Research Letters 33, L15106

Montgomery, D. \& Phillips, L. 1988, Phys. Rev. A 38, 2953

Qiu, J., Gary, D. E., \& Fleishman, G. D. 2009, Solar Phys., 255, 107

Titov, V. S., Hornig, G., \& Démoulin, P. 2002, J. Geophys. Res. 107, 1164

Wiegelmann, T. 2010, this volume 